\title{
Disentangling the relative merits and disadvantages of parentage analysis and assignment tests for inferring population connectivity
}

Authors: Mark R. Christie ${ }^{1,2, *}$, Patrick G. Meirmans ${ }^{3}$, Oscar E. Gaggiotti ${ }^{4}$, Robert J. Toonen $^{5}$, and Crow White 6

1. Department of Biological Sciences, Purdue University; 915 W. State St., West Lafayette, Indiana 47907-2054 USA

2. Department of Forestry and Natural Resources, Purdue University; 715 W. State St., West Lafayette, Indiana 47907-2054 USA

3. Institute for Biodiversity and Ecosystem Dynamics, University of Amsterdam, P.O. Box 94248, NL-1090 GE, Amsterdam, the Netherlands

4. Scottish Oceans Institute, University of St Andrews, East Sands, St Andrews, KY16 8LB, Fife, UK

5. Hawai'i Institute of Marine Biology, School of Ocean \& Earth Sciences \& Technology, University of Hawai'i at Mānoa, Kāne‘ohe, Hawai'i 96744 USA

6. Biological Sciences Department, California Polytechnic State University, San Luis Obispo, California 93407

*corresponding author: markchristie@ purdue.edu, phone: (765) 494-2070, fax: (765) 4940876 


\section{ABSTRACT:}

Accurately estimating patterns of population connectivity in marine systems remains an elusive goal. Current genetic approaches have focused on assigning individuals back to their natal populations using one of two methods: parentage analyses and assignment tests. Each of these approaches has their relative merits and weaknesses. Here, we illustrate these tradeoffs using a forward-time agent-based model that incorporates relevant natural history and physical oceanography for 135 Kellet's whelk (Kelletia kelletii) populations from Southern California. Like most marine organisms, Kellet's whelks live in large metapopulations where local populations are connected by dispersive larvae. For estimating population connectivity, we found parentage analyses to be relatively insensitive to the amount of genetic differentiation among local populations, but highly sensitive to the proportion of the metapopulation sampled. Assignment tests, on the other hand, were relatively insensitive to the proportion of the metapopulation sampled, but highly sensitive to the amount of genetic differentiation found among local populations. Comparisons between the true connectivity matrices (generated by using the true origin of all sampled individuals) and those obtained via parentage analyses and assignment tests reveal that neither approach can explain more than $26 \%$ of the variation in true connectivity. Furthermore, even with perfect assignment of all sampled individuals, sampling error alone can introduce noise into the estimated population connectivity matrix. Future work should aim to improve the number of correct assignments without the expense of additional incorrect assignments, perhaps by using dispersal information gleaned from related individuals as priors in a Bayesian framework. These analyses dispel a number of common misconceptions in the field and highlight areas for both future research and methodological improvements.

Key Words: larval connectivity, dispersal, marine metapopulations, genetic assignment tests, parentage analysis 


\section{INTRODUCTION:}

From which local population does the next generation originate? This fundamental question in marine biology remains unresolved, with significant consequences for advancing our understanding of ecological and evolutionary processes. Population connectivity at the generation or cohort scale is defined by contemporary rates of migration and includes the movement of indivdiuals among local populations (Botsford et al., 2009b; Burgess et al., 2014) often resulting in the formation of marine metapopulation structure (Kritzer \& Sale 2004). In coastal marine systems, patterns of population connectivity exhibited by fish, invertebrate, and algal species with a dispersive larval phase are spatially and temporally complex. These diverse patterns of larval connectivity can: $i$. promote persistence in unproductive "up-current" populations (Aiken and Navarrete, 2011), ii. shape demographic and genetic structure within marine metapopulations (Hedgecock and Pudovkin, 2011; Pringle et al., 2011; Watson et al., 2012; Williams and Hastings, 2013), iii. shift range boundaries (Gaylord and Gaines, 2000), iv. drive evolutionary change by characterizing the interactions between gene flow, genetic drift, and selection (Sanford and Kelly, 2011; De Wit and Palumbi, 2013), and $v$. enhance biodiversity by facilitating coexistence among competitor species (Chesson and Warner, 1981; Berkley et al., 2010; Edwards and Stachowicz, 2011). Furthermore, in this era of unprecedented anthropogenic impacts on marine ecosystems, accounting for connectivity can have substantial societal and economic value by enhancing conservation efficacy and boosting fisheries yields and profits (Costello et al., 1998; Botsford et al., 2009a; Christie et al., 2010; Truelove et al., 2015). For these reasons, quantifying population connectivity has been a major objective of marine science for over twenty years (Hixon 2011; Burgess et al., 2014).

While a number of approaches exist to estimate population connectivity in marine systems (reviewed by Hedgecock et al., 2007; Botsford et al., 2009b; Cowen and Sponaugle, 
2009; Pineda et al., 2009), the application of molecular genetics is among the more popular and fastest changing tools currently available for empirically quantifying marine connectivity. Motivated by the significant biological and socioeconomic implications for understanding contemporary patterns of connectivity, and fueled by rapid technological advances (Kool et al., 2013), connectivity research has recently moved beyond population genetics approaches for estimating average patterns of gene flow on evolutionary time scales, to those focused on identifying connections between populations with individual members of larval cohorts (e.g., Almany et al., 2013; D'Aloia et al., 2015). Different statistical approaches are available to measure connectivity using molecular markers such as hypervariable microsatellites or high-throughput sequencing to score single nucleotide polymorphisms (SNPs). These approaches can be divided into two general classes: 1. those that aim to assign individual recruits (successful larval settlers) back to their parents (hereafter: parentage analysis) and 2. those that aim to assign individual recruits back to their natal population (e.g., likelihood-based assignment tests, hereafter: assignment tests). Specifically, parentage analysis matches a recruit with a parent or parent-pair from a pool of candidate parents by resolving the Mendelian pattern of shared alleles between the parent and offspring (Peery et al., 2008; Planes and Lemer, 2011; Buston et al., 2012; Harrison et al., 2012; Christie, 2013), while assignment tests (as we define them here) match an individual recruit to its source population based on the expected frequency of its multi-locus genotype in alternative putative sources (Manel et al., 2005).

Individual-based genetic methods have revealed significant insights into the processes driving marine connectivity. For example, realized dispersal from management areas to local populations outside of their borders (larval seeding; Planes et al. 2009; Christie et al., 2010; Harrison et al. 2012), retention of larvae within a marine protected area (Berumen et al., 2012), localized recruitment of kin groups (Iacchei et al., 2013), surprising origins of long- 
distance colonists (Concepcion et al., 2016), correspondence of ocean currents with realized patterns of gene flow (White et al., 2010; Crandall et al., 2012), and hierarchical population structure in species with high gene flow (Knutsen et al., 2003; Benestan et al., 2015) have all been identified with individual-based genetic methods. Furthermore, in the most extensive parentage study undertaken to date, D'Aloia et al. (2015) generated an empirical dispersal kernel for larvae of the Caribbean goby, Elacatinus lori. They showed that despite an average pelagic larval duration (PLD) of $\sim 26$ days, the median dispersal distance of recruits was just $1.7 \mathrm{~km}$ and the maximum dispersal distance was $16.4 \mathrm{~km}$ (D'Aloia et al., 2015). Although extremely useful for describing general patterns, these methods must necessarily focus on relatively small areas that do not provide robust inferences about range-wide connectivity (i.e., estimates of migration rates between all populations across the species' range).

Accurate estimates of population connectivity throughout a species' range would substantially advance and support marine science and spatial management by providing a complete matrix of source-destination migration rates (Botsford et al., 2009b). However, in practice such information is rarely generated, even for a single focal location (for singular estuarine and amphidromous examples see Walther and Thorrold, 2008; and Hogan et al., 2014). This information gap is greatest for open-coast marine species (e.g., fishes and invertebrates living in kelp forests and coral reefs) due to their large population sizes, high rates of gene flow, and complex larval dispersal patterns (Kinlan and Gaines, 2003; Morgan and Fisher, 2010; Hare et al., 2011). For assignment tests, certainty of assignment of a recruit to its true source is typically low due to limited genetic differentiation between the true source and its alternatives, thereby increasing the chance of false assignment (Cornuet et al., 1999). For parentage analysis, large numbers of loci or analytical methods that focus on shared rare alleles substantially reduce the likelihood of false matches (Peery et al., 2008; Christie, 2013); however, only a small fraction of potential parents and offspring can be 
feasibly sampled. Additional sampling, analysis of more genetic data (both loci and alleles; Ryman et al., 2006), and enhanced statistics (e.g., matching recruits to unsampled populations, Duchesne et al., 2005; deciphering cryptic population structure, Manel et al., 2007) can help mitigate these challenges, but these solutions have logistical and/or analytical limits, and an overall understanding of the general tradeoffs in their efficacy and feasibility remains unknown. Consequently, for marine species the challenge lies in finding robust and cost-effective sampling designs and statistical approaches to individual-based genetic methods for achieving comprehensive, precise, and accurate estimates of connectivity.

Previous research comparing the results of parentage analysis and assignment tests using clownfish as a case study found assignment tests to be unreliable at differentiating between true and false sources of recruits in a well-mixed (high gene flow) metapopulation (SaenzAgudelo et al., 2009), corroborating theoretical evidence that assignment should be reliable only with sufficient population structuring (Cornuet et al., 1999). Other empirical research on Atlantic cod found assignment tests to be much more successful at determining recruit-source matches in a fairly well-mixed system, except between adjacent sources with extremely low genetic differentiation (Bradbury et al., 2011). Furthermore, assignment tests have proven valuable in identifying outside immigrants that presumably represent the tail end of the probability distribution of migration rates (Saenz-Agudelo et al., 2009; Concepcion et al., 2016). Parentage analysis, on the other hand, has proven successful within genetically homogenous metapopulations, but often has far fewer assignments (Saenz-Agudelo et al., 2009; Christie et al., 2010; Berumen et al., 2012; Pusack et al. 2014; D'Aloia et al., 2015; Salles et al., 2015).

Despite the recent advances in connectivity research, it is unlikely that there will be a single best approach for all types of marine metapopulations. For example, parentage studies 
typically focus on fishes with relatively low larval dispersal capabilities and a small area of each species' entire range containing relatively few, small populations that can be sampled extensively (reviewed by Riginos et al., 2011). Consideration of populations across the full range of an open-coast marine species, and a focus on species with high gene flow (e.g., via pelagic eggs or with longer PLDs) and large populations sizes (totaling in the hundreds of thousands to millions; Gomez-Uchida and Banks, 2006; Neilson, 2011; Simmonds et al., 2014), prohibits such complete sampling, resulting in far fewer expected successful parentoffspring matches. Thus, the number of parent-offspring pairs detected, and the ability for parentage analysis to detect immigrants, has been highly variable across studies (Peery et al., 2008; Christie et al., 2010; Saenz-Agudelo et al., 2011; D'Aloia et al., 2015). Additionally, not knowing the actual migration rates limits the ability to validate estimates of connectivity in empirical studies. To date, there has been no systematic evaluation of this problem with reference to known migration rates.

Here, we use a forward-time agent-based model of metapopulation dynamics that tracks the multi-locus genotypes of all adult, larval, and recruit individuals in space and time in order to explore the strengths and weaknesses of parentage analysis and assignment tests for studying patterns of larval connectivity in open-coast marine species under realistic sampling scenarios. We perform a number of simulations to generate known migration matrices and then compare the results from parentage analysis and assignment tests to better understand the relative benefits and limitations of each approach for resolving population connectivity in marine systems. 


\section{METHODS:}

\section{Simulation model}

To systematically evaluate the performance of assignment tests and parentage methods for estimating population connectivity, we developed a spatially-explicit, forward-time, agentbased model $(\mathrm{ABM})$. The model was built using relevant life-history information from the subtidal marine gastropod Kellet's whelk (Kelletia kelletii) in the Pacific Ocean off the coast of Southern California (Fig. 1). Kellet's whelk share many life history characteristics with typical nearshore marine fish and invertebrate species: large population sizes; slow-growing, sedentary, demersal adults; and pelagic larvae (Allen et al., 2006). Specifically, Kellet's whelk have a dispersive larval stage with a pelagic larval duration of 40-60 days (Romero et al., 2012), after which the larvae settle to rocky substrate in the subtidal zone where they remain highly site attached for the duration of their lives (Rosenthal, 1970; Zacherl et al., 2003). Because many marine species are characterized by large population sizes with many local populations and large ranges (e.g., Gomez-Uchida and Banks, 2006; Neilson, 2011), we modeled an entire metapopulation consisting of 135 local populations distributed throughout Southern California. Furthermore, the physical oceanography of this region has been extensively characterized, such that we were able to obtain species-specific connectivity matrices from a biophysical model of regional ocean circulation (Mitarai et al., 2009; described below).

Each local population in the ABM was characterized by an average of 1,000 individuals, resulting in an average metapopulation size of 135,000 adult whelks that approximates the lower end of an empirical estimate of the species' population size in the study region (95\% CI: 82,615-1,556,300; Simmonds et al., 2014). Each individual was randomly assigned a sex (male or female) and was characterized by 500 independent single nucleotide polymorphisms (SNPs). We focused on SNPs because they have been shown to 
provide high statistical power for both assignment tests and parentage analysis (Anderson and Garza 2006; Narum et al., 2008; Hauser et al., 2011) although analyses using microsatellite markers would be expected to yield similar results (Liu et al., 2005; Glover et al., 2010). At the beginning of each model run, all multi-locus genotypes were created in accordance with Hardy-Weinberg Equilibrium (HWE) with a minor allele frequency of 0.2. After initializing populations, the $\mathrm{ABM}$ was characterized by the following steps: mortality, reproduction, larval dispersal, and recruitment (Fig. 1). We assumed an average 20\% mortality rate per year, following estimates for coastal marine species (Lee et al., 2011; Rassweiler et al., 2012). This process created age-structured populations with overlapping generations and a mean generation time of 4.8 years. During the mortality step, individuals were randomly removed from throughout the metapopulation without respect to local population. Within each local population, mortality rates were varied slightly each year using a random deviate from a normal distribution with a mean equal to the number of needed offspring and a standard deviation of 10 . This process increased the fluctuations in population sizes among local populations and mimics marine population dynamics (Fig. S1).

For computational efficiency (i.e., memory limitations), we only created as many offspring as needed to keep the metapopulation size constant through time. Thus, we only created offspring that would survive through the settlement stage. Because many marine organisms are characterized by high variance in reproductive success (Hedgecock and Pudovkin, 2011), we varied the number of offspring produced by each pair (with most pairs producing no offspring in a given year) using a gamma distribution with a shape parameter of 0.5 and a rate parameter of 0.1 . Pairs were created by randomly pairing males and females within each local population. Offspring were created in strict accordance with Mendelian inheritance; at each locus, each offspring inherited one allele, chosen at random, from both parents. 


\section{Population connectivity}

To simulate larval (i.e., offspring) dispersal among the 135 local populations, we used data from a biophysical model developed by Mitarai et al. (2009) built upon an ocean circulation model (OCM) of southern California and a particle tracking model (PTM). The OCM is a high-resolution Regional Ocean Modeling System (ROMS) of the study region driven by realistic boundary conditions (See Supporting Information for more details). Larval connectivity was quantified using the Lagrangian probability density function (PDF) method (Mitarai et al., 2009) that estimates connectivity from a source site to a destination site by quantifying the probability of particle displacement over a specified time period. To incorporate the larval life history of Kellet's whelk, the particles were released from June through August, the spawning period for Kellet's whelk (Zacherl et al., 2003), and tracked for 40 to 60 dispersal days, the PLD of Kellet's whelk larvae (Romero et al., 2012). Lagrangian PDFs were obtained for each spawning month and dispersal day for 1996-2002, the years available from Mitarai et al. (2009). The PDFs were averaged across spawning months, dispersal days and then years to generate a mean connectivity matrix (See Supplementary Table 1 for actual values). Using this connectivity matrix, which resulted in high rates of gene flow, the equilibrium value of global $F_{S T}$ was equal to 0 .

We applied the above connectivity matrix to each local population in the $\mathrm{ABM}$ to determine the number of recruits originating from each of the 135 local populations. While using this connectivity matrix resulted in high levels of connectivity and gene flow, rates of self-recruitment were not insubstantial (see inset of Fig. 1 for region-wide connectivity matrix and Supplementary Table 1 for actual values). To specifically determine the number of offspring to create in each source population, we used a multinomial distribution specifying all 135 populations, the number of needed offspring for a local population, and the connectivity matrix describing the probability of a recruit originating from each of $K$ local 
populations. In practice, the multinomial distribution was implemented prior to reproduction so that we knew precisely how many offspring to create in each local population, which increased computational efficiency. However, the actual dispersal of individuals occurred after reproduction, as illustrated in Fig. 1.

\section{Levels of differentiation}

Because previous work has illustrated that the performance of assignment tests can vary for different levels of genetic differentiation, we created metapopulations with three levels of genetic differentiation: high, medium, and low. Here, we measured genetic differentiation as $F_{S T}$ (calculated using the equation of Weir and Cockerham, 1984; and implemented in GENODIVE and HIERFSTAT; Meirmans and Van Tienderen, 2004, Goudet 2005) and we created marine metapopulations with high $\left(F_{S T}=0.1\right)$, medium $\left(F_{S T}=0.01\right)$, and low $\left(F_{S T}=\right.$ 0.001) levels of genetic differentiation among all local populations (sensu Palumbi 2003). To create these three values, we first ran each simulation for 100 years using a connectivity matrix with $100 \%$ self-recruitment, such that there was no gene flow among local populations; this process, combined with the naturally high variance in reproductive success among adults (see model details above), allowed for local populations to experience genetic drift such that genetic differentiation increased through time (each population was initiated with identical allele frequencies). After 100 years, we switched connectivity matrices to the empirical matrix generated by the OCM and PTM in relation to Kellet's whelk life history, and continued to run the model forward in time until the model reached the desired level of global genetic differentiation. This approach necessitates that the local populations were not in drift-migration equilibrium upon sampling, but empirical evidence suggests that relatively few marine populations are in such a state of equilibrium (Hellberg 2009; Hart and Marko 2010; Wares 2010). Furthermore, both the parentage and assignment methods do not make the assumption of drift-migration equilibrium. 
For each level of genetic differentiation $\left(F_{S T}=0.001,0.01\right.$ and 0.1$)$, we created 30 independent replicates, and sampled between 10 and 100 adults (in increments of 10) and an equal number of recruits from each of 20 populations spaced equidistantly throughout the seascape. We chose 20 populations and a maximum sample of size of 100 adults and 100 recruits at each site (study-wide sample size of 4,000 individuals) because in practice this sample size represents a considerable amount of field and laboratory effort and expense. These values are also reasonable given our metapopulation size of 135,000 individuals and translate into sampling between 0.3 to 3 percent of the entire metapopulation. Because many marine metapopulations are in the range of at least several hundred-thousand individuals (Gomez-Uchida and Banks, 2006; Neilson, 2011; Simmonds et al., 2014), these values capture the sampling challenges faced when studying many marine organisms. With 30 independent replicates for each of 3 levels of genetic differentiation and 10 different sample sizes, we obtained a total of 900 data sets that were each subsequently analyzed with parentage analysis and assignment tests. Subsequent analyses (parentage and assignment) were performed independently and without knowledge of the simulation outcome to avoid any potential bias. All simulations were run in R version 3.2 (R Core Team 2016) on a high performance computing cluster where each node had 20 cores (dual 10-core Intel(R) Xeon(R) E5-2660 v3 CPU), with 256 GB memory per node.

\section{Parentage analysis}

Parentage analyses were performed with scripts modified from the software SOLOMON (Christie et al., 2013). Here, we examined all 500 SNP loci and performed simple Mendelian exclusion. Similar results could be obtained by using smaller numbers of loci, particularly for data sets with smaller sample sizes, or by accounting for the frequencies of shared alleles (Christie, 2010). For each individual in the model, the birth population and adult population were recorded. This feature allowed us to compare the assignments of recruits to adults using 
the multi-locus parentage analyses to the true dispersal pathway and thus calculate the number of correct and incorrect assignments (Type I errors; Christie, 2010). There were no Type II errors, i.e., true parent-offspring pairs that were included in a sample but not identified as such, because we did not introduce any genotyping errors into the model, such that all parent-offspring pairs shared at least one allele at each of the 500 loci.

\section{Assignment tests}

Assignment tests were performed using the original likelihood-based approach from Paetkau et al. (1995), in combination with Monte Carlo simulations to calculate significance (Cornuet et al., 1999; Paetkau et al., 2004). For every sampled recruit, the multilocus likelihood was calculated for each of the 20 sampled populations, following Paetkau et al. (1995). For each population, we first calculated the observed allele frequencies in the sampled adults. Next, the likelihood of the diploid recruit genotype originating from each local population was calculated based on the adult allele frequencies, assuming Hardy Weinberg equilibrium in the populations. Multilocus likelihoods over all 500 loci were obtained by taking the product of the single-locus likelihoods. Because population-specific allele frequencies of zero at a single locus will lead to biased multilocus likelihoods of zero, we substituted all allele frequencies of zero with a value of $5 \times 10^{-3}$. This value has been shown to give the best results in a test of six different replacement values (Paetkau et al., 2004). We did not use the partial Bayesian method from Rannala \& Mountain (1997), which does not require such a replacement value and generally shows better performance (Cornuet et al. 1999, Hauser et al. 2006) because there is no software implementation of that method that can be automated to analyze a large numbers of datasets. For every recruit, the population with the highest likelihood was taken as the inferred source population and compared to the known source population (as reported in Figs. 3A \& B). Because not all true source populations were sampled (mimicking realistic 
sampling scenarios; see details above) any migrant born in an unsampled population was assigned 'incorrectly' in addition to any errors made by the method itself.

To account for the bias caused by incomplete sampling of source populations, we also used Monte Carlo simulations to generate null distributions for the assignments of the recruits (Cornuet et al., 1999; Paetkau et al., 2004). Two possible test statistics can be used for this approach. The first test statistic is $L_{h}$; the likelihood with which the recruit originated in the population from which it was sampled. The second test statistic is the ratio $L_{h} / L_{\max }$, where $L_{\max }$ is the maximum likelihood over all sampled populations. We used the $L_{h}$ test statistic to generate Figs. 3C \& D because this statistic is more suitable when only a small percentage of possible source populations have been sampled (Paetkau et al., 2004), which is the case for most studies attempting to estimate marine population connectivity. However, the drawback of using $L_{h}$ is that it only distinguishes between migrants and non-migrants ("residents" i.e., individuals recruited back to their natal populations). Thus, this approach cannot be used to build connectivity matrices, but may still be useful for estimating self-recruitment. In the Monte Carlo simulations, new datasets were formed by randomly creating individual genotypes by randomly drawing gametes from the genotypes observed in the sampled adults. This method of drawing whole gametes from the observed individuals was previously found to give much better results than the alternative method of creating individuals by drawing random alleles based on the observed allele frequencies (Paetkau et al., 2004). For every replicate dataset of adults and recruits that was tested, 100 Monte Carlo datasets were created. This means that, depending on the sample size of the data set, 20,000 to 200,000 genotypes were created for calculating the null distribution of the $L_{h}$-statistic. For every recruit, a $p$ value was calculated by comparing the observed $L_{h}$-statistic to the generated null-distribution. Using an alpha of 0.01, we considered assignments correct if individuals with a p-value $\leq$ 0.01 were spawned in a different local population than where they were sampled and we 
considered assignments incorrect if an individual with a $p$-value $\leq 0.01$ was spawned in the sampled population. An alternative to the Monte-Carlo test is to calculate, for every individual, the contribution of every population to the sum of the likelihoods of all sampled populations (Piry et al. 2004). High values for one particular population would provide evidence that the individual was truly from that population, whereas low values for all populations would mean that the true source population was not included. Using this method, we found that the great majority of individuals had a value very close to one for the population with the maximum likelihood, including individuals dispersing from unsampled populations (up to $87 \%$ had a value $>0.95$, depending on the value of $F_{\text {ST }}$ and the sample size). Therefore, we could not use this method to further distinguish true migrants from false positives. The assignments and Monte Carlo permutations were performed using a pre-release version of the software GENODIVE 3.0 (Meirmans and Van Tienderen, 2004).

\section{Quantifying method performance}

To compare population connectivity matrices for all correct assignments of recruits within a sample to those generated by either parentage analyses or assignment tests, we calculated the fraction of unexplained variance (FUV; Simons et al., 2013). This parameter was calculated as:

$$
F U V=1-\operatorname{cor}(\mathbf{A}, \mathbf{B})^{2}
$$

where, for a given data set, $\mathbf{A}$ is the "true" connectivity matrix generated with the correct assignment of all sampled recruits, $\mathbf{B}$ is the connectivity matrix determined via parentage analyses or assignment tests and cor equals Pearson's correlation between the two matrices. Note, for $\mathbf{A}$ and $\mathbf{B}$ to be comparable, the matrices were limited to describing the rates of connectivity for recruits from the 20 sampled populations. Thus, the matrices are 20x20 and 
here we use realized dispersal matrices (Watson et al. 2010), where each matrix sums to one and represents the relative contribution of pair-wise connectivity to system-wide recruitment.

\section{RESULTS:}

Results from the parentage analyses revealed that the proportion of sampled recruits matched successfully to a parent increases as the fraction of the metapopulation sampled increased (Fig. 2). Stated differently, the absolute number of parent-offspring pairs identified in a sample increased with the total number of individuals sampled (Fig. S2). Because we used 500 SNP loci scored without genotyping error, the number of unrelated individuals falsely identified as a parent-offspring pair was negligible for our sample sizes (less than 1 false assignment per data set: Fig. 2b). The amount of genetic differentiation between populations did not change either the number of correct or incorrect parent-offspring pairs identified.

By contrast, results from assignment approaches were strongly influenced by genetic differentiation (Fig. 3). Using the highest likelihood score for each recruit, correct assignments were highest when $F_{S T}$ was high $\left(\sim 14 \%\right.$ correctly assigned when $\left.F_{S T}=0.1\right)$ and lowest when genetic differentiation was low ( $4 \%$ correctly assigned when $\left.F_{S T}=0.001\right)$ (Fig. 3A). Also in contrast to parentage methods, this approach was relatively insensitive to sample sizes; larger sample sizes did not yield more assignments that were correct. Because assignment approaches attempt to assign every individual back to the most likely source population from which data exists (see Methods), the percentage of incorrect assignments was very high. Therefore, the proportion of incorrect assignments was inflated by individuals that originated in a population that was not sampled (all individuals spawned in an unsampled population must result in an incorrect assignment because their natal population is not among the choices to which they can be assigned). Even for $F_{S T}$ values that are high by marine population standards $\left(F_{S T}=0.1\right.$; the highest $F_{S T}$ we tested $)$, the percentage of recruits that were incorrectly assigned was greater than $80 \%$ (Fig. 3B), but it is also important to keep in 
mind that only $15 \%$ of true source populations were sampled (i.e., 20/135 sites), and could therefore be assigned correctly, in this design. To address this bias, we also employed the $L_{h}$ approach, which classifies individuals as being either migrants or not migrants (i.e., spawned in and recruited to the sampled population). Using this approach, the percentage of incorrect assignments (i.e., residents falsely classified as migrants) decreased to $<1 \%$ (alpha $=0.01$; Fig. 3D). This means that almost all recruits that were tagged as migrants, using the $L_{h}$ approach and an alpha of 0.01 , were indeed migrants. However, the reverse is not true; not all recruits with $p>0.01$ were residents. It is noteworthy that this approach shows a nonintuitive relationship with sample size when $F_{S T}=0.1$, where larger sample sizes resulted in slightly fewer correct assignments on average than seen at lower sample sizes, but with tighter confidence intervals (Fig. 3C). Regardless of the sample size, the proportion of correct assignments was higher using assignment than parentage approaches, and nearly an order of magnitude higher when using the $L_{h}$ approach and $F_{S T}=0.1$ (Fig. 3C). However, the $L_{h}$ approach illustrated in Figure 3C-D can only identify individuals as being immigrants, and cannot identify the source population from which an immigrant originated; thus, this approach cannot be used to build connectivity matrices. Another caveat is that in real studies the type II error rate (i.e., the number of immigrants not identified as immigrants) will remain unknown, such that using this approach may over-estimate rates of self-recruitment as individuals with p-values greater than alpha cannot be assumed to be residents. However, given that caveat, this approach may still be useful to provide a maximum estimate of selfrecruitment given the low rates of incorrect assignment (provided that appropriate caveats and power-analyses are provided) and, perhaps more usefully, can identify a lower bound on rates of immigration even if the actual populations that are exchanging migrants cannot be identified. 
We next converted our parentage and assignment test results into connectivity matrices, and compared the results with their corresponding true connectivity matrices visually and using fraction of unexplained variance ( $F U V$; eq. 1 in Methods). It is important to note, that by examining the assignment of settled recruits, our matrices are recruit-centered and retrospective. That is, for the recruits that arrived at a particular site, the matrices indicate the proportion that came from other sites. This type of connectivity could be combined with estimates of offspring production to estimate rates of demographic connectivity. We first illustrate results for a hypothetical empirical scenario (using two independent replicates; one illustrated in Fig. 4 and the other in Fig. S3), where we assume every single recruit could be sampled and assigned successfully back to its natal population (Fig. 4A). Under this hypothetical scenario, we found a considerable amount of self-recruitment, particularly between sites 15 to 60 , which mimics the underlying empirical dispersal matrix used to simulate connectivity in the ABM ( $c f$. Fig. 4A with Fig. 1 inset). We next sampled half of the recruits at each of our 20 sampling sites and allowed for perfect assignment. We found that there is considerable noise introduced into the connectivity matrix when half of the total number of recruits is sampled at a local population even when every single recruit is assigned correctly (Fig. 4B). This result suggests that even with perfect assignment, limited sample sizes will reduce the accuracy of empirically derived population connectivity matrices. By comparison, a connectivity matrix derived from the same replicate data (half of all recruits sampled at all 20 sites), but using parentage analysis clearly revealed the primary limitation of this method: a very sparse matrix is generated (Fig. 4C). Furthermore, even though every parent-offspring pair represents a correct assignment, the parentage assignments do not always correspond with the regions of greatest population connectivity. Lastly, an example using the highest likelihood score revealed the primary limitation of assignment tests: large numbers of incorrect assignments result in a connectivity matrix that suggests that a 
disproportionately large number of recruits were spawned in population 36 (Fig. 4D). The large number of incorrect assignments to population 36 was not driven by that population being more genetically differentiated than the other sampled populations (Fig. S4).

To examine the sensitivity of the accuracy of parentage analyses and assignment tests for estimating connectivity in relation to experimental design and population genetic differentiation we calculated $F U V$ between true and estimated connectivity matrices across sample sizes and for each of the three levels of $F_{S T}($ Fig. 5). For parentage analysis, there was no substantial effect of genetic differentiation on the fraction of unexplained variance. However, unexplained variance decreased as the proportion of the metapopulation sampled increased. For assignment tests, $F U V$ decreased with increasing genetic differentiation (i.e., performance was better for assignment tests with higher $F_{S T}$ values). For low and moderate levels of $F_{S T}$, there was a slight reduction in $F U V$ for greater proportions of the metapopulation sampled. At higher proportions of the metapopulation sampled, parentage analyses outperformed assignment tests at estimating population connectivity matrices, with parentage analyses accounting for up to $26 \%$ of the variance explained and assignment tests accounting for up to $5 \%$ of the variance explained. However, for the realistic sample and metapopulation sizes examined in this study, both approaches leave a substantial portion of the variance unexplained.

\section{DISCUSSION:}

A significant challenge in marine ecology is the delineation of populations of individuals and an understanding of the barriers or linkages that determine the evolutionary trajectory among these biological entities (Waples and Gaggiotti, 2006). Thus, defining populations and quantifying exchange among those populations is central to the fields of ecology, evolution, and conservation biology. Yet the fundamental question of where do the next generation of 
local recruits originate remains unresolved in most highly dispersive species (Botsford et al., 2009b; Burgess et al., 2014). This knowledge gap underscores the considerable logistical and statistical challenges to quantifying connectivity in the marine environment. For example, at the quadrennial International Coral Reef Symposium (ICRS) in June 2016 only a single study (D'Aloia et al., 2015) attempted to estimate a complete matrix of source-destination migration rates between pairwise populations throughout the study domain, and even this ambitious study did not attempt to do so across the entirety of the species' range. Further, nearly $90 \%$ of presentations at ICRS with the stated goal of estimating connectivity employed parentage analyses rather than assignment tests. Although this is admittedly a narrow sample of coralreef studies, it is our impression that such uneven distribution of one of these two approaches is common in marine science, and seems to be reflected in reviews and grant proposals that we have seen in recent years as well. Notably, in fisheries science assignment and genetic stock identification methods (GSI, a method closely related to assignment as defined here; Ihssen et al., 1981; Carvalho and Hauser, 1994) are predominant (Hauser and Carvalho 2008), whereas in marine ecology research parentage analysis seems to predominate (e.g., Jones et al., 2009; Kool et al., 2013), at least currently. Despite numerous assertions in the literature (e.g., Botsford et al., 2009b; Kool et al., 2013) and strong opinions expressed by colleagues about which approach is superior, we know of no simulation-based quantitative analysis where both approaches could be compared with known connectivity matrices (but see: Hauser et al. 2006 and Saenz-Agudelo et al. 2009 for empirical approaches).

\section{Relative strengths and weaknesses of parentage and assignment approaches}

A primary difference between the approaches is that, with so many SNPs, parentage provides very high quality of matches between recruits and their source locations (Fig. 2B), but the number of parent-offspring pairs identified is a small fraction of the total individuals genotyped (Fig. 2A). In contrast, assignment testing can estimate a source population for 
every recruit, because all recruits are assigned regardless of statistical confidence, but the quality of those assignments is much lower (Fig 3A), and the number of incorrect assignments can be substantial, especially with low $F_{S T}$ values (Fig 3B; Manel et al. 2005). However, it is only possible to assign recruits to the correct origin if the source population has been sampled (Slatkin 2005), so by definition every individual originating from an unsampled population is incorrectly assigned (recall that only $15 \%$ of the sources were sampled). The result is a large proportion (>80\%, Fig. 3B) of incorrect assignments in assignment testing relative to parentage analyses. For parentage analysis, individuals from unsampled adults cannot be assigned and are thus not counted in the incorrect assignments category (provided sufficient numbers of loci are used); however, the overall number of assigned individuals is far lower $(<5 \%$, Fig. $2 \mathrm{~A})$. As one way to account for the high rate of false assignment with assignment tests, we also compared results of the Monte Carlo test where p-values tag individual recruits as being migrants. In this case, the proportion of selfrecruiting individuals being falsely tagged as migrants decreases dramatically to be on par with parentage (Fig. 3D). Further, the proportion of sampled recruits that are correctly tagged as migrants is at the least comparable with parentage analysis, and can be nearly an order of magnitude greater when population structure is moderately high $\left(F_{S T}>0.01\right.$; Fig. 3C). However, whereas almost all recruits tagged as migrants were indeed migrants, there were still a lot of migrants not tagged as such, even at $F_{S T}=0.1$. Thus, researchers should not make the mistake of interpreting the non-significant $p$-values, as these represent a mixture of both residents and migrants.

So just tell me, which method should I use?

We wish there was a simple answer to this question. As outlined in the previous section, the most fundamental difference between these two approaches is a tradeoff between the quality and quantity of matching recruits to source locations. Essentially parentage has relatively 
few, but very high quality assignments that are robust to underlying population genetic structure, but are highly sensitive to the proportion of the population sampled. On the other hand, assignment approaches connect every individual to a source population, but with low average quality of assignments that are relatively robust to sample size, but are highly sensitive to the underlying population genetic structure.

Thus, for estimating population connectivity, parentage analysis is a logical choice so long as the experimental design of sampling is scaled appropriately to the size of the study population (as asserted by, e.g., Jones et al., 2009). Consequently, if samples can be collected non-lethally or from fisheries by-catch, the value of this method is limited only by logistical and financial constraints to ensure that a sufficient proportion of both recruits and potential parents are sampled (the ideal design is a 50/50 split; Christie, 2013) and an estimate of the proportion of correct assignments can be obtained by taking the product of the proportion of parents sampled and the proportion of recruits sampled. Our results indicate that the increase in the proportion of correctly assigned individuals is directly proportional to sample size of the total population, and to avoid generating sparse connectivity matrices, the simple conclusion is the larger the sample size the better. With the continuing decrease in sequencing costs, it may soon be possible to affordably genotype tens of thousands of individuals. Thus, for parentage analysis, a primary limitation will not be in the genotyping of individuals, but rather in logistical and financial constraints associated with collecting tissue samples from a large number of individuals. In terms of practical limitations, sampling as many adults and offspring as possible is the best approach, but genotyping errors, fewer loci, or greater numbers of pair-wise comparisons could potentially increase the number of incorrect assignments to parent-offspring pairs, such that we strongly advocate performing $a$ priori power analyses before embarking upon parentage studies (see Christie, 2013). Furthermore, the relative insensitivity of parentage to the degree of population structure 
leaves parentage as a practical option for species with very low genetic population differentiation provided that enough loci are obtained to employ exclusion-based methods (i.e., methods that do not rely upon estimates of allele frequencies as was performed in this paper). If parentage methods that rely on allele frequency estimates are required, then it is important to choose methods that can account for these differences without bias (e.g., see Figure 2 of Christie 2010; Anderson and Weir 2007).

On the other hand, assignment tests can perform well when $F_{S T}$ is moderate to high (> 0.01). Thus, in terms of minimizing incorrect assignments, parentage analyses will perform best in systems with little to no genetic differentiation (if using methods that estimate allele frequencies) and assignment tests will perform best in systems with moderate to high genetic differentiation. Further, it is noteworthy that when there is high population structure, the vast majority of incorrect population assignments come from individuals originating from unsampled populations. Thus, assignment is a logical choice for situations in which the sample size is constrained below the fraction needed to fill in a connectivity matrix using parentage and the number of potential source populations is limited. This may be particularly applicable for open coast marine species with massive population sizes where sampling will necessarily be proportionally small, provided there is sufficient genetic structure to differentiate among populations (as asserted by, e.g., Manel et al., 2005; Botsford et al., 2009b). To reduce unexplained variation, the simple conclusion is that sampling from as many potential sources as possible will minimize the bias towards incorrect assignment. In terms of practical limitations, the relative insensitivity of the method to sample size effects suggests that the most effective sampling design would be to sample only as many individuals as needed to get an estimate of the allele frequencies from every possible source population throughout the species range (Cornuet et al. 1999; Ruzzante 1998; Kalinowski 2005; Hale et al. 2012). For many previously unstudied populations, it may be most effective 
to perform small pilot studies to determine $i$. the best method to select, ii. the type of sampling scheme to employ on a large scale, and iii. whether the questions of interest can be answered sufficiently with the chosen species.

\section{Shortcomings of both approaches and future directions}

As outlined in the sections above, neither approach currently provides an ideal solution to trying to generate a complete matrix of population connectivity. In fact, our results indicate that neither method is currently well-suited to estimate quantitative migration rates. Instead, they only provide evidence of connectivity between populations but at such low precision relative to the truth that they are effectively qualitative rather than quantitative. For example, in cases where no recruits in a focal population can be matched with parents elsewhere does not imply a complete lack of connectivity between sites; it may simply be that the sample size and/or number of sites sampled was not sufficiently large to capture the true underlying connectivity pattern. Without an explicit incorporation of uncertainty into such measurements, we currently have no way to evaluate quantitative estimates of migration in natural populations that rely on these approaches.

Further, these conclusions hold true even if all sampled recruits could be successfully assigned back to their natal populations. As highlighted in Figure 4, simply by incorporating some sampling error (50\% of recruits sampled and assigned with 100\% accuracy - a relatively small level of sampling error compared to that expected in any empirical study) the resultant connectivity matrix becomes highly divergent from the true underlying pattern of connectivity ( $c f$. Fig. 4A, B). When we apply a realistic sampling scheme of up to 4,000 individuals, we find that neither the parentage nor the assignment test approach produces a connectivity matrix resembling the underlying truth (Fig. 5). Surprisingly, neither approach can explain more than $26 \%$ of the variation between the estimated connectivity matrices and 
the underlying truth. This is a discouraging result that highlights the considerable need for additional work and future improvement in these methods. Nevertheless, when a larger percentage of the metapopulation was sampled, parentage analyses outperformed assignment tests (Fig. 5) because sparse, but mostly correct matrices can better recover the true connectivity matrix than complete, but mostly incorrect matrices (Fig. S5). These differences between the two methods disappear as parentage-based methods become increasingly sparse (i.e., less of the metapopulation is sampled) or as assignment tests result in fewer incorrect assignments (e.g., more of the metapopulation is sampled). One important caveat is that we used a connectivity matrix that was fairly complex, being derived from oceanographic models (see Methods; Figure 1). In some marine systems, however, it is possible that the patterns of connectivity are not only much more simple, but also constant through time. In these systems, the fraction of unexplained variance may be considerably lower for both parentage methods and population assignment. On the other hand, differences in local population sizes and oceanographic conditions may create vastly different connectivity matrices from year to year such that, for those systems, we may be underestimating the fraction of unexplained variance.

Many argue that there are very good reasons to pursue a fully-resolved connectivity matrix for conservation and sustainable, productive fisheries management (e.g., Botsford et al., 2009a; Costello et al., 2010). While we see avenues to approach this goal (see below), our results suggest that neither method is currently up to the task for the majority of open coast marine species. On the other hand, it may be sufficient to simply identify the relative extent to which marine populations are open or closed (e.g., Cowen et al., 2000; Mora and Sale, 2002). The degree to which marine systems are open or closed has long been debated (e.g., Thorson, 1950; Scheltem, 1971; Crisp, 1976), and is a question of great relevance to evolutionary biology, population and spatial ecology, management, and conservation. These 
questions may be answered using alternative approaches such as identifying statistical relationships between dispersal strength and distance (i.e., dispersal kernels), incorporating demography into estimates of local retention, or focusing on specific pairwise connections (e.g., MPAs). On a more positive note, our results show that the $L_{h}$ approach (Cornuet et al., 1999; Paetkau et al., 2004) performs quite well to distinguish migrants from non-migrants and provides a reliable method by which to determine the degree to which a population is open or closed. However, we also caution that the answer to this question is likely to vary in both space and time (e.g., Toonen and Grosberg, 2011), thus patterns of connectivity need to be estimated over multiple years to fully understand the spatial and temporal dynamics of marine population connectivity. Along these lines, an additional useful approach may be to look for complementary insights between single generation genetic approaches and population genetic approaches that account for many generations of gene flow.

Moving forward, the most productive avenues for advancement would be to focus on combining methods analytically for enhanced certainty. Two approaches, which are not mutually exclusive, that hold particular promise include: $i$. combining parentage and assignment methods to leverage the strengths of each (e.g., use identified parent-offspring pairs as priors to assignment probabilities in a Bayesian approach); and $i$. combining genetic with independent non-genetic methods of assignment to reduce uncertainty in the combined analyses (e.g., oceanographic models and microchemistry analysis results to serve as priors for genetic approaches to estimating connectivity rates; Levin, 2006; Botsford et al., 2009b; Jones et al., 2009; Leis et al., 2011). In such cases, the hurdle that needs to be overcome is to explicitly calculate uncertainty in each approach and use those uncertainties to provide quantitative estimates of migration with known confidence (Palsboll et al., 2010). While there currently is no ideal method to estimate full connectivity matrices in large marine populations, we see advancing the early efforts to explicitly combine approaches (e.g., 
Gaggiotti et al., 2002; Gaggiotti et al., 2004; Miller et al., 2005; Smith and Campana, 2010; Simmonds et al., 2014) as the best approach to harness the power of each and take advantage of the relative strengths of one approach to offset the relative weaknesses of another.

\section{ACKNOWLEDGEMENTS:}

We thank Satoshi Mitarai for assistance generating the larval dispersal connectivity matrix and Darren Johnson for providing valuable feedback on an earlier draft of this manuscript. We also thank the anonymous reviewers and the editor for providing comments that have greatly improved this manuscript. This research was supported in part through computational resources provided by Information Technology at Purdue, West Lafayette, Indiana. This work was also supported in part by NSF OCE 12-60169 to RJT, and RJT, OEG and PGM were supported by the 2015 Pauley Summer Program at the Hawai'i Institute of Marine Biology where this collaborative project began. OEG was also supported by MASTS (the Marine Alliance for Science and Technology for Scotland). 


\section{FIGURE LEGENDS:}

Figure 1: Illustration of steps in the forward-time agent-based models. Populations were modeled after the southern California coastline and consisted of 135 local populations of Kellet's whelk (numbers shown on map). Each local population consisted of approximately 1000 individuals, where each individual was characterized by 500 SNP loci. Steps in the model consisted of density-dependent mortality (e.g., competition, predation), densityindependent mortality (e.g., variability in environmental conditions), reproduction, and dispersal, where dispersal was modeled using the connectivity matrix from a biophysical model parameterized with realistic ocean current and life history conditions (see Methods). For dispersal, we illustrate the connectivity matrix used for all 135 local populations where the color scale ranges from blue (low connectivity) to red (high connectivity).

Figure 2: Percentage of correct and incorrect assignments for parentage analyses for different proportions of the metapopulation sampled (mean $\pm 95 \% \mathrm{CI}$ ). A correct assignment consisted of a true parent-offspring pair being identified as such. An incorrect assignment consisted of an unrelated pair of individuals being classified incorrectly as a parent-offspring pair. In all cases, the same 20 equally spaced populations were sampled at different sampling intensities (see text for details). For parentage analysis, the percentage of correct assignments increased with sample sizes. With the 500 loci, there were almost no false assignments (type I error) and there were no true pairs that remained undetected (type II error). Points with no visible error bars had confidence intervals that were smaller than the diameter of the point. Notice that correct assignments are not affected by genetic differentiation and scale nearly linearly with the percent of the entire metapopulation sampled.

Figure 3: Percentage of correct and incorrect assignments for assignments tests (mean $\pm 95 \%$ CI). Panels A and B show results using assignment of all recruits based on the best likelihood 
score for each individual. Notice that assignment methods perform better for metapopulations with greater levels of genetic differentiation and also that these methods assign all individuals such that the number of correct and incorrect assignments sum to $100 \%$ of recruits sampled. Panels $\mathrm{C}$ and $\mathrm{D}$ show results using only the $L_{h}$ test statistic to assign individuals as migrants (i.e., born in a different population than where they were sampled) based on individuals that had p-values less than or equal to 0.01 (see text). It is important to note that in panels C and D individuals identified as migrants cannot be assigned to their specific source (i.e., natal) population, but this approach may be useful for estimating self-recruitment. Points with no visible error bars had confidence intervals that were smaller than the diameter of the point.

Figure 4: Estimated connectivity matrices, illustrated as heatmaps, for 4 scenarios: A. Every recruit in a local population was sampled and correctly assigned (i.e., we used their known origin). B. Half of all recruits in a local population were sampled, but all still correctly assigned (introduction of sampling error). Notice that even with $100 \%$ correct assignment (for which no empirical method exists), substantial noise is generated by simply not sampling every individual. C. Half of all recruits sampled (as in B), but where the connectivity matrix was generated from identified parent-offspring pairs, D. Half of all recruits sampled (as in B), but where the connectivity matrix was generated with the best likelihood score from the assignment tests $\left(F_{S T}=0.1\right.$ for all scenarios $)$. All matrices are examples from a single representative replicate (see Fig. 5 for a broader examination of the parameter space).

Figure 5: Fraction of unexplained variance for parentage analysis and assignment tests as a function of both sample sizes and population differentiation (mean $\pm 95 \% \mathrm{CI}$ ). Upper plots represent parentage analysis for $F_{S T}$ values of A) 0.1 ; B) 0.01 ; C) 0.001 , each across a fraction of the metapopulation sampled from 0.3 to $3.0 \%$. Lower plots represent assignment tests with best likelihood scores for $F_{S T}$ values of D) 0.1 ; E) 0.01 ; F) 0.001 . Parentage and 
assignment-test derived connectivity matrices were compared to scenarios where all recruits from the sample were correctly assigned. 


\section{REFERENCES:}

Aiken, C. M., and Navarrete, S. A. 2011. Environmental fluctuations and asymmetrical dispersal: generalized stability theory for studying metapopulation persistence and marine protected areas. Marine Ecology-Progress Series, 428: 77-88.

Allen, L. G., Pondella II, D. J., and Horn, M. H. 2006. The Ecology of Marine Fishes: California and Adjacent Waters, University of California, Los Angeles. 660 pp.

Almany, G. R., Hamilton, R. J., Bode, M., Matawai, M., Potuku, T., Saenz-Agudelo, P., Planes, S., et al. 2013. Dispersal of grouper larvae drives local resource sharing in a coral reef fishery. Current Biology, 23: 626-630.

Anderson, E. C., and Garza, J.C.. 2006. The power of single-nucleotide polymorphisms for large-scale parentage inference. Genetics 172: 2567-2582.

Anderson, A.D., and Weir, B.S. 2007. A maximum-likelihood method for the estimation of pairwise relatedness in structured populations. Genetics 176: 421-440.

Benestan, L., Gosselin, T., Perrier, C., Sainte-Marie, B., Rochette, R., and Bernatchez, L. 2015. RAD genotyping reveals fine-scale genetic structuring and provides powerful population assignment in a widely distributed marine species, the American lobster (Homarus americanus). Molecular Ecology, 24: 3299-3315.

Berkley, H. A., Kendall, B. E., Mitarai, S., and Siegel, D. A. 2010. Turbulent dispersal promotes species coexistence. Ecology Letters, 13: 360-371.

Berumen, M. L., Almany, G. R., Planes, S., Jones, G. P., Saenz-Agudelo, P., and Thorrold, S. R. 2012. Persistence of self-recruitment and patterns of larval connectivity in a marine protected area network. Ecology and Evolution, 2: 444-452.

Botsford, L. W., Brumbaugh, D. R., Grimes, C., Kellner, J. B., Largier, J., O'Farrell, M. R., Ralston, S., et al. 2009a. Connectivity, sustainability, and yield: bridging the gap between conventional fisheries management and marine protected areas. Reviews in Fish Biology and Fisheries, 19: 69-95.

Botsford, L. W., White, J. W., Coffroth, M. A., Paris, C. B., Planes, S., Shearer, T. L., Thorrold, S. R., et al. 2009b. Connectivity and resilience of coral reef metapopulations in marine protected areas: matching empirical efforts to predictive needs. Coral Reefs, 28: 327-337.

Bradbury, I. R., Hubert, S., Higgins, B., Bowman, S., Paterson, I. G., Snelgrove, P. V. R., Morris, C. J., et al. 2011. Evaluating SNP ascertainment bias and its impact on population assignment in Atlantic cod, Gadus morhua. Molecular Ecology Resources, 11: 218-225.

Burgess, S. C., Nickols, K. J., Griesemer, C. D., Barnett, L. A. K., Dedrick, A. G., Satterthwaite, E. V., Yamane, L., et al. 2014. Beyond connectivity: how empirical methods can quantify population persistence to improve marine protected-area design. Ecological Applications, 24: 257-270.

Buston, P. M., Jones, G. P., Planes, S., and Thorrold, S. R. 2012. Probability of successful larval dispersal declines fivefold over $1 \mathrm{~km}$ in a coral reef fish. Proceedings of the Royal Society B-Biological Sciences, 279: 1883-1888.

Carvalho, G. R., and Hauser, L. 1994. Molecular-Genetics and the Stock Concept in Fisheries. Reviews in Fish Biology and Fisheries, 4: 326-350. 
Chesson, P. L., and Warner, R. R. 1981. Environmental Variability Promotes Coexistence in Lottery Competitive-Systems. American Naturalist, 117: 923-943.

Christie, M. R. 2010. Parentage in natural populations: novel methods to detect parentoffspring pairs in large data sets. Molecular Ecology Resources, 10: 115-128.

Christie, M. R. 2013. Bayesian parentage analysis reliably controls the number of false assignments in natural populations. Molecular Ecology, 22: 5731-5737.

Christie, M. R., Tennessen, J. A., and Blouin, M. S. 2013. Bayesian parentage analysis with systematic accountability of genotyping error, missing data and false matching. Bioinformatics, 29: 725-732.

Christie, M. R., Tissot, B. N., Albins, M. A., Beets, J. P., Jia, Y. L., Ortiz, D. M., Thompson, S. E., et al. 2010. Larval Connectivity in an Effective Network of Marine Protected Areas. Plos One, 5.

Concepcion, G. T., Kenyon, J., Baums, I. B., and Toonen, R. J. 2016. Genetic evidence for possible coral larval dispersal from the Northern Line Islands to the Hawaiian Archipelago. Galaxea, Journal of Coral Reef Studies, 18: 7-9.

Cornuet, J. M., Piry, S., Luikart, G., Estoup, A., and Solignac, M. 1999. New methods employing multilocus genotypes to select or exclude populations as origins of individuals. Genetics, 153: 1989-2000.

Costello, C., Rassweiler, A., Siegel, D., De Leo, G., Micheli, F., and Rosenberg, A. 2010. The value of spatial information in MPA network design. Proceedings of the National Academy of Sciences of the United States of America, 107: 18294-18299.

Costello, C. J., Adams, R. M., and Polasky, S. 1998. The value of El Nino forecasts in the management of salmon: A stochastic dynamic assessment. American Journal of Agricultural Economics, 80: 765-777.

Cowen, R. K., Gawarkiewic, G., Pineda, J., Thorrold, S. R., and Werner, F. E. 2007. Population Connectivity in Marine Systems An Overview. Oceanography, 20: 14-21.

Cowen, R. K., Lwiza, K. M. M., Sponaugle, S., Paris, C. B., and Olson, D. B. 2000. Connectivity of marine populations: Open or closed? Science, 287: 857-859.

Cowen, R. K., and Sponaugle, S. 2009. Larval Dispersal and Marine Population Connectivity. Annual Review of Marine Science, 1: 443-466.

Crisp, D. J. 1976. The role of the pelagic larva. Perspectives in experimental biology, 1: 145155.

D'Aloia, C. C., Bogdanowicz, S. M., Francis, R. K., Majoris, J. E., Harrison, R. G., and Buston, P. M. 2015. Patterns, causes, and consequences of marine larval dispersal. Proceedings of the National Academy of Sciences of the United States of America, 112: $13940-13945$.

De Wit, P., and Palumbi, S. R. 2013. Transcriptome-wide polymorphisms of red abalone (Haliotis rufescens) reveal patterns of gene flow and local adaptation. Molecular Ecology, 22: 2884-2897.

Duchesne, P., Castric, T., and Bernatchez, L. 2005. PASOS (parental allocation of singles in open systems): a computer program for individual parental allocation with missing parents. Molecular Ecology Notes, 5: 701-704. 
Edwards, K. F., and Stachowicz, J. J. 2011. Spatially stochastic settlement and the coexistence of benthic marine animals. Ecology, 92: 1094-1103.

Gaggiotti, O. E., Brooks, S. P., Amos, W., and Harwood, J. 2004. Combining demographic, environmental and genetic data to test hypotheses about colonization events in metapopulations. Molecular Ecology, 13: 811-825.

Gaggiotti, O. E., Jones, F., Lee, W. M., Amos, W., Harwood, J., and Nichols, R. A. 2002. Patterns of colonization in a metapopulation of grey seals. Nature, 416: 424-427.

Gaylord, B., and Gaines, S. D. 2000. Temperature or transport? Range limits in marine species mediated solely by flow. American Naturalist, 155: 769-789.

Gilg, M. R., and Hilbish, T. J. 2003. The geography of marine larval dispersal: Coupling genetics with fine-scale physical oceanography. Ecology, 84: 2989-2998.

Glover, K. A., Hansen, M. M., Lien, S., Als, T. D., Hoyheim, B., and Skaala, O. 2010. A comparison of SNP and STR loci for delineating population structure and performing individual genetic assignment. BMC Genetics, 11.

Gomez-Uchida, D., and Banks, M. A. 2006. Estimation of effective population size for the long-lived darkblotched rockfish Sebastes crameri. Journal of Heredity, 97: 603-606.

Goudet, Jérôme. 2005. Hierfstat, a package for R to compute and test hierarchical F-statistics. Molecular Ecology Notes 5: 184-186

Grantham, B. A., Eckert, G. L., and Shanks, A. L. 2003. Dispersal potential of marine invertebrates in diverse habitats. Ecological Applications, 13: S108-S116.

Hale, M.L., Burg T.M., and Steeves, T.E. 2012. Sampling for microsatellite-based population genetic studies: 25 to 30 individuals per population is enough to accurately estimate allele frequencies. PloS one 7.9: e45170.

Hare, M. P., Nunney, L., Schwartz, M. K., Ruzzante, D. E., Burford, M., Waples, R. S., Ruegg, K., et al. 2011. Understanding and Estimating Effective Population Size for Practical Application in Marine Species Management. Conservation Biology, 25: 438449.

Harrison, H. B., Williamson, D. H., Evans, R. D., Almany, G. R., Thorrold, S. R., Russ, G. R., Feldheim, K. A., et al. 2012. Larval Export from Marine Reserves and the Recruitment Benefit for Fish and Fisheries. Current Biology, 22: 1023-1028.

Hauser L., Seamons T.R., Dauer M., Naish K.A.,and Quinn T.P. 2006. An empirical verification of population assignment methods by marking and parentage data: hatchery and wild steelhead (Oncorhynchus mykiss) in Forks Creek, Washington, USA. Molecular Ecology 15: 3157-3173.

Hauser, L., and Carvalho, G.R. 2008. Paradigm shifts in marine fisheries genetics: ugly hypotheses slain by beautiful facts. Fish and Fisheries 9: 333-362.

Hauser, L., Baird, M., Hilborn, R., Seeb, L. W., and Seeb, J. E. 2011. An empirical comparison of SNPs and microsatellites for parentage and kinship assignment in a wild sockeye salmon (Oncorhynchus nerka) population. Molecular Ecology Resources, 11: 150-161.

Hedgecock, D., Barber, P. H., and Edmands, S. 2007. Genetic Approaches to Measuring Connectivity. Oceanography, 20: 70-79. 
Hedgecock, D., and Pudovkin, A. I. 2011. Sweepstakes Reproductive Success in Highly Fecund Marine Fish and Shellfish: A Review and Commentary. Bulletin of Marine Science, 87: 971-1002.

Hixon, M. A. 2011. 60 Years of Coral Reef Fish Ecology: Past, Present, Future. Bulletin of Marine Science, 87: 727-765.

Iacchei, M., Ben-Horin, T., Selkoe, K. A., Bird, C. E., Garcia-Rodriguez, F. J., and Toonen, R. J. 2013. Combined analyses of kinship and FST suggest potential drivers of chaotic genetic patchiness in high gene-flow populations. Molecular Ecology, 22: 3476-3494.

Ihssen, P. E., Booke, H. E., Casselman, J. M., Mcglade, J. M., Payne, N. R., and Utter, F. M. 1981. Stock Identification - Materials and Methods. Canadian Journal of Fisheries and Aquatic Sciences, 38: 1838-1855.

Jones, G. P., Almany, G. R., Russ, G. R., Sale, P. F., Steneck, R. S., van Oppen, M. J. H., and Willis, B. L. 2009. Larval retention and connectivity among populations of corals and reef fishes: history, advances and challenges. Coral Reefs, 28: 307-325.

Kalinowski, S.T. 2005. Do polymorphic loci require large sample sizes to estimate genetic distances? Heredity 94: 33-36.

Kinlan, B. P., and Gaines, S. D. 2003. Propagule dispersal in marine and terrestrial environments: A community perspective. Ecology, 84: 2007-2020.

Knutsen, H., Jorde, P. E., Andre, C., and Stenseth, N. C. 2003. Fine-scaled geographical population structuring in a highly mobile marine species: the Atlantic cod. Molecular Ecology, 12: 385-394.

Kool, J. T., Moilanen, A., and Treml, E. A. 2013. Population connectivity: recent advances and new perspectives. Landscape Ecology, 28: 165-185.

Kritzer, J. P., and Sale, P.F. 2004. Metapopulation ecology in the sea: from Levins' model to marine ecology and fisheries science. Fish and Fisheries 5: 131-140.

Lee, H. H., Maunder, M. N., Piner, K. R., and Methot, R. D. 2011. Estimating natural mortality within a fisheries stock assessment model: An evaluation using simulation analysis based on twelve stock assessments. Fisheries Research, 109: 89-94.

Leis, J. M., Van Herwerden, L., and Patterson, H. M. 2011. Estimating Connectivity in Marine Fish Populations: What Works Best? Oceanography and Marine Biology: An Annual Review, Vol 49, 49: 193-234.

Levin, L. A. 2006. Recent progress in understanding larval dispersal: new directions and digressions. Integrative and Comparative Biology, 46: 282-297.

Liu, N. J., Chen, L., Wang, S., Oh, C. G., and Zhao, H. Y. 2005. Comparison of singlenucleotide polymorphisms and microsatellites in inference of population structure. Bmc Genetics, 6.

Manel, S., Berthoud, F., Bellemain, E., Gaudeul, M., Luikart, G., Swenson, J. E., Waits, L. P., et al. 2007. A new individual-based spatial approach for identifying genetic discontinuities in natural populations. Molecular Ecology, 16: 2031-2043.

Manel, S., Gaggiotti, O. E., and Waples, R. S. 2005. Assignment methods: matching biological questions techniques with appropriate. Trends in Ecology \& Evolution, 20: 136-142. 
Meirmans, P. G., and Van Tienderen, P. H. 2004. GENOTYPE and GENODIVE: two programs for the analysis of genetic diversity of asexual organisms. Molecular Ecology Notes, 4: 792-794.

Miller, J. A., Banks, M. A., Gomez-Uchida, D., and Shanks, A. L. 2005. A comparison of population structure in black rockfish (Sebastes melanops) as determined with otolith microchemistry and microsatellite DNA. Canadian Journal of Fisheries and Aquatic Sciences, 62: 2189-2198.

Mitarai, S., Siegel, D. A., Watson, J. R., Dong, C., and McWilliams, J. C. 2009. Quantifying connectivity in the coastal ocean with application to the Southern California Bight. Journal of Geophysical Research-Oceans, 114.

Mora, C., and Sale, P. F. 2002. Are populations of coral reef fish open or closed? Trends in Ecology \& Evolution, 17: 422-428.

Morgan, S. G., and Fisher, J. L. 2010. Larval behavior regulates nearshore retention and offshore migration in an upwelling shadow and along the open coast. Marine Ecology Progress Series, 404: 109-126.

Narum, S. R., Banks, M., Beacham, T. D., Bellinger, M. R., Campbell, M. R., Dekoning, J., Elz, A., et al. 2008. Differentiating salmon populations at broad and fine geographical scales with microsatellites and single nucleotide polymorphisms. Molecular Ecology, 17: 3464-3477.

Neilson, D. J. 2011. Assessment of the California Spiny Lobster (Panulirus interruptus) California Department of Fish and Game, San Diego, CA, USA. 138 pp.

Ohlmann, J. C., and Mitarai, S. 2010. Lagrangian assessment of simulated surface current dispersion in the coastal ocean. Geophysical Research Letters, 37.

Paetkau, D., Calvert, W., Stirling, I., and Strobeck, C. 1995. Microsatellite Analysis of Population-Structure in Canadian Polar Bears. Molecular Ecology, 4: 347-354.

Paetkau, D., Slade, R., Burden, M., and Estoup, A. 2004. Genetic assignment methods for the direct, real-time estimation of migration rate: a simulation-based exploration of accuracy and power. Molecular Ecology, 13: 55-65.

Palsboll, P. J., Peery, M. Z., and Berube, M. 2010. Detecting populations in the 'ambiguous' zone: kinship-based estimation of population structure at low genetic divergence. Molecular Ecology Resources, 10: 797-805.

Palumbi, S. R. 2003. Population genetics, demographic connectivity, and the design of marine reserves. Ecological Applications, 13: S146-S158.

Peery, M. Z., Beissinger, S. R., House, R. F., Berube, M., Hall, L. A., Sellas, A., and Palsboll, P. J. 2008. Characterizing Source-Sink Dynamics with Genetic Parentage Assignments. Ecology, 89: 2746-2759.

Pineda, J., Reyns, N., and Starczak, V. 2009. Complexity and simplification in understanding recruitment in benthic populations. Population Ecology, 51: 17-32.

Piry, S., Alapetite, A., Cornuet, J.-M., Paetkau, D., Baudouin, L. \& Estoup, A. 2004 GENECLASS2: a software for genetic assignment and first-generation migrant detection. Journal of Heredity, 95: 536-539.

Planes, S., Jones, G.P., and Thorrold, S.R. 2009. Larval dispersal connects fish populations in a network of marine protected areas. Proceedings of the National Academy of Sciences 106: 5693-5697. 
Planes, S., and Lemer, S. 2011. Individual-based analysis opens new insights into understanding population structure and animal behaviour. Molecular Ecology, 20: 187-189.

Pringle, J. M., Blakeslee, A. M. H., Byers, J. E., and Roman, J. 2011. Asymmetric dispersal allows an upstream region to control population structure throughout a species' range. Proceedings of the National Academy of Sciences of the United States of America, 108: $15288-15293$.

Rannala, B. \& Mountain, J. 1997. Detecting immigration by using multilocus genotypes. Proceedings of the National Academy of Sciences of the United States of America 94: 9197-9201.

Pusack, T.J., Christie, M.R., Johnson, D.W., Stallings, C.D., Hixon, M.A. 2014 Spatial and temporal patterns of larval dispersal in a coral-reef fish metapopulation: evidence of variable reproductive success. Molecular Ecology 23: 3396-3408.

Rassweiler, A., Costello, C., and Siegel, D. A. 2012. Marine protected areas and the value of spatially optimized fishery management. Proceedings of the National Academy of Sciences of the United States of America, 109: 11884-11889.

Riginos, C., Douglas, K. E., Jin, Y., Shanahan, D. F., and Treml, E. A. 2011. Effects of geography and life history traits on genetic differentiation in benthic marine fishes. Ecography, 34: 566-575.

Romero, M. R., Walker, K. M., Cortez, C. J., Sanchez, Y., Nelson, K. J., Ortega, D. C., Smick, S. L., et al. 2012. Larval diel vertical migration of the marine gastropod Kelletia kelletii (Forbes, 1850). Journal of Marine Biology, 2012.

Rosenthal, R. 1970. Observations on the reproductive biology of the Kellet's Whelk, Kelletia kelletii. Veliger, 12: 319-324.

Ruzzante, D.E. 1998. A comparison of several measures of genetic distance and population structure with microsatellite data: bias and sampling variance. Canadian Journal of Fisheries and Aquatic Sciences 55: 1-14.

Ryman, N., Palm, S., Andre, C., Carvalho, G. R., Dahlgren, T. G., Jorde, P. E., Laikre, L., et al. 2006. Power for detecting genetic divergence: differences between statistical methods and marker loci. Molecular Ecology, 15: 2031-2045.

Saenz-Agudelo, P., Jones, G. P., Thorrold, S. R., and Planes, S. 2009. Estimating connectivity in marine populations: an empirical evaluation of assignment tests and parentage analysis under different gene flow scenarios. Molecular Ecology, 18: 17651776.

Saenz-Agudelo, P., Jones, G. P., Thorrold, S. R., and Planes, S. 2011. Connectivity dominates larval replenishment in a coastal reef fish metapopulation. Proceedings of the Royal Society B-Biological Sciences, 278: 2954-2961.

Salles, O. C., Maynard, J. A., Joannides, M., Barbu, C. M., Saenz-Agudelo, P., Almany, G. R., Berumen, M. L., et al. 2015. Coral reef fish populations can persist without immigration. Proceedings of the Royal Society B-Biological Sciences, 282.

Sanford, E., and Kelly, M. W. 2011. Local Adaptation in Marine Invertebrates. Annual Review of Marine Science, Vol 3, 3: 509-535. 
Scheltem, R. S. 1971. Larval Dispersal as a Means of Genetic Exchange between Geographically Separated Populations of Shallow-Water Benthic Marine Gastropods. Biological Bulletin, 140: 284-\&.

Simmonds, S. E., Kinlan, B. P., White, C., Paradis, G. L., Warner, R. R., and Zacherl, D. C. 2014. Geospatial statistics strengthen the ability of natural geochemical tags to estimate range-wide population connectivity in marine species. Marine Ecology Progress Series, 508: 33-51.

Simons, R. D., Siegel, D. A., and Brown, K. S. 2013. Model sensitivity and robustness in the estimation of larval transport: A study of particle tracking parameters. Journal of Marine Systems, 119: 19-29.

Slatkin, M. 2005. Seeing ghosts: the effect of unsampled populations on migration rates estimated for sampled populations. Molecular Ecology, 14: 67-73.

Smith, S. J., and Campana, S. E. 2010. Integrated stock mixture analysis for continous and categorical data, with application to genetic-otolith combinations. Canadian Journal of Fisheries and Aquatic Sciences, 67: 1533-1548.

Thorson, G. 1950. Reproductive and larval ecology of marine bottom invertebrates. Biological Reviews of the Cambridge Philosophical Society, 25: 1-45.

Toonen, R. J., and Grosberg, R. K. 2011. Causes of chaos: spatial and temporal genetic heterogeneity in the intertidal anomuran crab Petrolisthes cinctipes. In Phylogeography and Population Genetics in Crustacea, pp. 75-107. Ed. by S. Koenemann, C. Held, and C. Schubard. CRC Press Crustacean Issues Series.

Truelove, N. K., Griffiths, S., Ley-Cooper, K., Azueta, J., Majil, I., Box, S. J., Behringer, D. C., et al. 2015. Genetic evidence from the spiny lobster fishery supports international cooperation among Central American marine protected areas. Conservation Genetics, 16: 347-358.

Waples, R. S., and Gaggiotti, O. 2006. What is a population? An empirical evaluation of some genetic methods for identifying the number of gene pools and their degree of connectivity. Molecular Ecology, 15: 1419-1439.

Watson, J. R., Mitarai, S., Siegel, D. A., Caselle, J.E., Dong, C., and McWilliams J.C. 2010. Realized and potential larval connectivity in the Southern California Bight. Marine Ecology Progress Series, 401: 31-48.

Watson, J. R., Kendall, B. E., Siegel, D. A., and Mitarai, S. 2012. Changing Seascapes, Stochastic Connectivity, and Marine Metapopulation Dynamics. American Naturalist, 180: 99-112.

Weersing, K., and Toonen, R. J. 2009. Population genetics, larval dispersal, and connectivity in marine systems. Marine Ecology Progress Series, 393: 1-12.

Weir, B. S., and Cockerham, C. C. 1984. Estimating F-Statistics for the Analysis of Population-Structure. Evolution, 38: 1358-1370.

Williams, P. D., and Hastings, A. 2013. Stochastic Dispersal and Population Persistence in Marine Organisms. American Naturalist, 182: 271-282.

Zacherl, D., Gaines, S. D., and Lonhart, S. I. 2003. The limits to biogeographical distributions: insights from the northward range extension of the marine snail, Kelletia kelletii (Forbes, 1852). Journal of Biogeography, 30: 913-924. 